Check for updates

Cite this: RSC Adv., 2019, 9, 9962

\title{
Zeolitic imidazolate framework-67 for shape stabilization and enhanced thermal stability of paraffin-based phase change materials $\dagger$
}

\author{
Yiwen Hu, (D)* Xiuduo Song, Qilong Zheng, Jiangning Wang and Jiangfei Pei
}

Zeolitic imidazolate framework-67 (ZIF-67), a new kind of metal-organic framework, has large surface area as well as outstanding thermal and chemical stability. In this paper, micro-sized ZIF-67 crystals were prepared and further employed as the reinforcing material to design novel paraffin-based composite phase change materials (PCMs) with a polymethyl methacrylate (PMMA) shell. The composite PCMs were fabricated by using a ZIF-67 crystal-stabilized oil-in-water (O/W) Pickering emulsion as a template. Morphologies and thermal properties of the prepared composite PCMs with different contents of ZIF-67 crystals were determined by SEM, DSC and TGA. Results showed that the ZIF-67 concentration in the emulsion system has a significant effect on the microstructure, phase change behavior and thermal stability of the resultant composite PCMs. When adding $1.5 \mathrm{~g}$ of ZIF-67 crystals, the resultant composite PCMs achieved a stable sphere-like structure and had about $106.06 \mathrm{~J} \mathrm{~g}^{-1}$ of latent heat. The prepared composite PCMs also exhibited a good thermal stability. Compared with pure paraffin, the thermostability of the shape-stabilized paraffin was significantly enhanced at a low content of ZIF-67 crystals.

Received 1st February 2019

DOI: $10.1039 / c 9 r a 00874 h$

rsc.li/rsc-advances

\section{Introduction}

In recent years, phase change materials (PCMs) have been extensively studied in thermal energy storage for their ability of absorbing or releasing a large amount of heat in their phase transition process..$^{1-4}$ Paraffin waxes are definitely a popular PCM since they have the advantages of high latent heat, are chemically inert, non-toxic, cheap and have adjustable phase change temperatures. ${ }^{4-6}$

In general, the use of bulk PCMs in thermal energy storage requires some special latent heat devices, resulting in the increase of economic costs. More importantly, the liquid state of PCMs is liable to leak and then diffuse throughout other materials. ${ }^{7,8}$ Therefore, many researchers recently focused on fabricating the shape-stable paraffin by employing porous materials as supporting matrix or encapsulate paraffin in a variety of polymeric shells. ${ }^{9-11}$ For example, Li et al..$^{9}$ designed a form-stable diatomite/paraffin composite PCMs by attracting paraffin into the nanopore of diatomite. Results showed that shape-stabilized paraffin has a significant improvement in thermal stability and thermal conductivity. Al-Shannaq et al. ${ }^{10}$ exploited a suspension polymerization method for encapsulating of paraffin with a polymethyl methacrylate (PMMA) shell.

Xi'an Modern Chemistry Research Institute, Xi'an, 710065, Shaanxi, P. R. China. E-mail: huyiwenn123@163.com

$\dagger$ Electronic supplementary information (ESI) available. See DOI: $10.1039 / \mathrm{c} 9 \mathrm{ra} 00874 \mathrm{~h}$
Fayyad et al. ${ }^{11}$ prepared microencapsulated paraffin with a ureaformaldehyde shell by in situ polymerization. As a result, microencapsulated paraffin could significantly increase the heat transfer area. However, the incorporation content of active paraffin in shape-stabilized composites needs to be improved for maximizing energy storage capacity, while the commonly used polymeric shells have some shortages such as poor mechanical property and low thermal stability. Thus, exploring novel materials and applying effective routes to design high performance composite PCMs are vital for the development of thermal energy storage technology.

Metal-organic frameworks (MOFs), a unique class of crystalline hybrid microporous materials, constructed from the assembling of metal ions or clusters with suitable organic linkers. ${ }^{12-14}$ Due to the excellent high specific surface areas, many experimental researches have been performed for exploring applications of MOFs, especially for gas storage or separation, photocatalysis. ${ }^{15,16}$ Zeolitic imidazolate frameworks67 (ZIF-67) is a novel kind of MOFs, which composed of the tetrahedrally coordinated divalent $\mathrm{Co}^{2+}$ metal ions and imidazolate ligands. It has highly chemical, mechanical, and thermal stability compared to general MOFs. ${ }^{1718}$ Recently, combining ZIFs with others special features of materials has been proposed and applied in diverse applications. Considering the shortages of the shape-stabilized and microencapsulated PCMs, ZIF-67 with high thermal stability makes it as an excellent material to stabilize or encapsulate PCMs for preventing their leakage and improving the thermal properties simultaneously. In this 
paper, we report for the first time to employ ZIF-67 as reinforcing material for the preparation of shape-stabilized composite PCMs.

Pickering emulsion is an emulsion stabilized by using small colloidal particles alone. ${ }^{19,20}$ Many reports have proved that it is feasible to prepare various Pickering emulsions by using MOF particle as stabilizer. ${ }^{21-23}$ Meanwhile, Pickering emulsion is a simple, reliable, and eco-friendly method to further obtain composites. $^{\text {20,24,25 }}$ Herein, a series of novel composite PCMs based on paraffin as core and ZIF-67/PMMA as hybrid shell were prepared by Pickering emulsion route. Micro-sized ZIF-67 crystals acted as stabilizer replacing traditional organic emulsifiers that inevitably existed in products and may produce some adverse effects on their performances. ZIF-67 crystals were first dispersed in aqueous phase. Then, an oil-in-water Pickering emulsion based on melted paraffin, monomer and initiator as oil phase was generated by a high-speed shear instrument. The subsequent polymerization reaction lead to the formed polymer chains separating from paraffin and depositing on the interface of ZIF-67 crystals oil phase (Fig. 1). Finally, the formed PMMA/ZIFs composites served as a protective layer imparting the PCMs good shape stability and thermal properties for thermal energy storage application. The effects of the loading contents of ZIF-67 crystals on the micromorphology, phase change behaviours and thermal stability of the paraffin/PMMA/ ZIF-67 composite PCMs were also investigated in details.

\section{Experimental}

\subsection{Materials}

Paraffin (50-54 $\left.{ }^{\circ} \mathrm{C}\right)$, 2-methylimidazole (99\%) and cobalt chloride hexahydrate $\left(\mathrm{Co}\left(\mathrm{NO}_{3}\right)_{2} \cdot 6 \mathrm{H}_{2} \mathrm{O}, 99.99 \%\right)$ were obtained from Aladdin Reagent Co., Ltd. Ethylene glycol dimethacrylate (PEGMA, 98\%, Xi'an Chemical Reagent Co., Ltd) was used as crosslinking agents and used without further purification. 2,2Azobisisobutyronitrile (AIBN, 98.5\%, Xi'an Chemical Reagent Co., Ltd) was employed as initiator. The solvents of methanol, ethanol and deionized water were purchased from Xi'an Chemical Reagent Co., Ltd. Prior to use, methyl methacrylate (MMA, 98\%, Xi'an Chemical Reagent Co., Ltd) was purified through a column of basic alumina to remove the inhibitor.

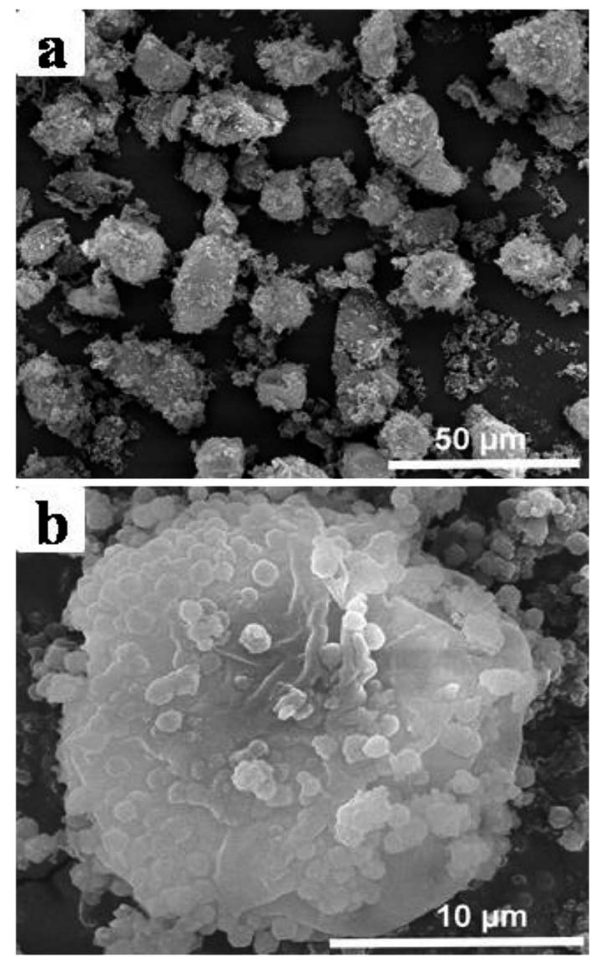

Fig. 2 SEM images of the synthesized paraffin/PMMA/ZIF-67 composite PCMs (ZPCMs-3).

\subsection{Preparation}

Micro-sized ZIF-67 crystals were synthesized according to a previous literature after a minor modification. ${ }^{26}$ In a typical process, 2-methylimidazole $(2.0525 \mathrm{~g}, 25 \mathrm{mmol})$ in $40 \mathrm{ml}$ methanol was gradually added into a solution of $1.455 \mathrm{~g}$ of $\mathrm{Co}\left(\mathrm{NO}_{3}\right)_{2} \cdot 6 \mathrm{H}_{2} \mathrm{O}(5 \mathrm{mmol})$ in $40 \mathrm{ml}$ methanol with stirring over $10 \mathrm{~min}$. Subsequently, the reaction mixture was transferred into a $100 \mathrm{ml}$ Teflon-lined autoclave and then placed it in a $120{ }^{\circ} \mathrm{C}$ for $24 \mathrm{~h}$. Finally, the products were collected by centrifuging and washed thoroughly with deionized water and ethanol.

Paraffin/PMMA/ZIF-67 composite PCMs were prepared by a Pickering emulsion method, in which ZIF-67 crystals served as single-emulsifier. Firstly, a certain amount of ZIF-67 crystals was ultrasonically dispersed into $100 \mathrm{ml}$ deionized water.

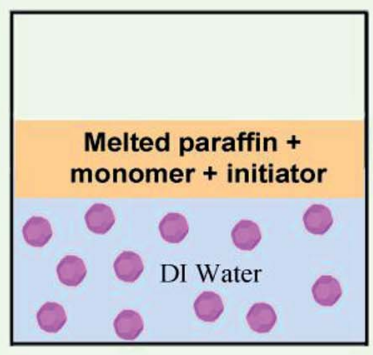

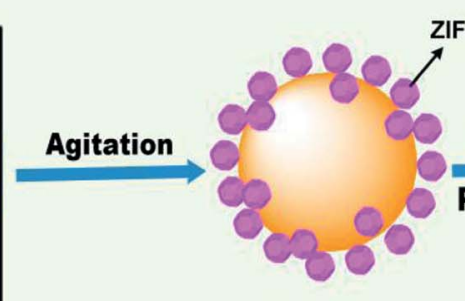

Oil droplet stabilized by ZIF-67

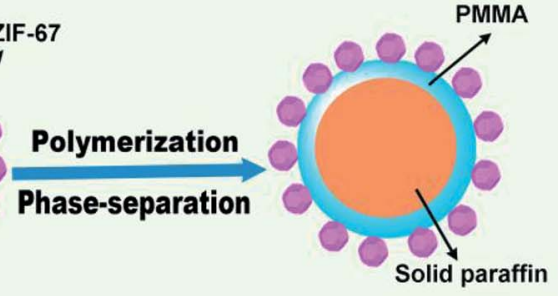

Composite PCM

Fig. 1 Schematic for the preparation of paraffin/PMMA/ZIF-67 composite PCMs. 


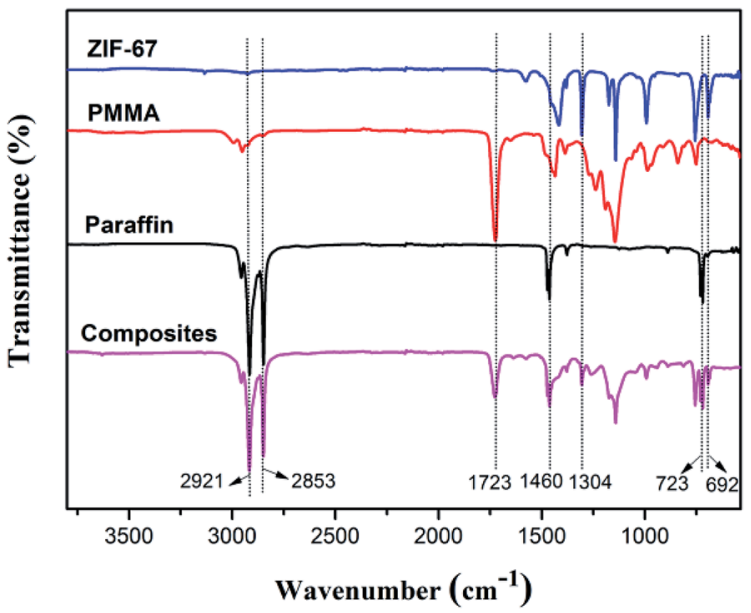

Fig. 3 FT-IR spectra of ZIF-67 crystals, PMMA, paraffin and composite PCMs (ZPCMs-3).

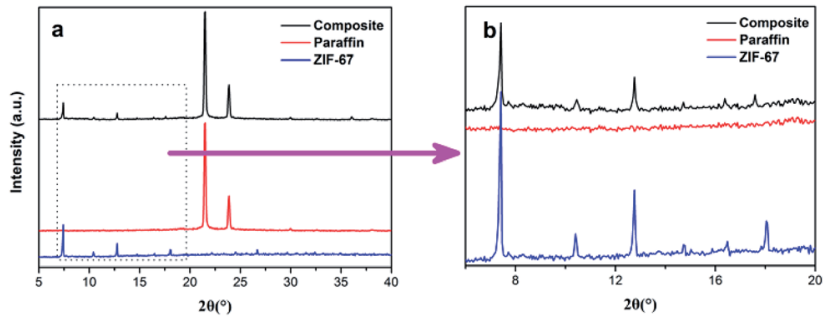

Fig. 4 XRD patterns of paraffin, ZIF-67, and composite PCMs (ZPCMs3).

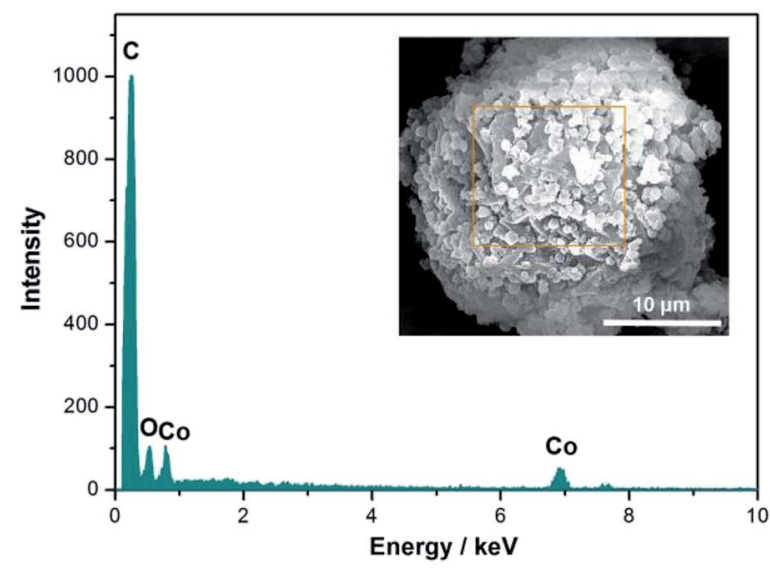

Fig. 5 EDX spectrum of the paraffin/PMMA/ZIF-67 composite PCMs (ZPCMs-3).

Meanwhile, $0.12 \mathrm{~g}$ of AIBN was dissolved in the mixture of MMA $(2 \mathrm{~g})$, PEGMA ( $2 \mathrm{~g})$ and melted paraffin $(6 \mathrm{~g})$. Then, mixed the above mixture with ZIF-67 dispersion in a $60{ }^{\circ} \mathrm{C}$ water bath, followed by agitate the mixture at $12000 \mathrm{rpm}$ for $3 \mathrm{~min}$ to form a stable Pickering emulsion. Afterwards, the resulting emulsion was transferred into $200 \mathrm{ml}$ flask and stirred with continuously agitating at $75{ }^{\circ} \mathrm{C}$ for $3.5 \mathrm{~h}$ in $\mathrm{N}_{2}$. Finally, the obtained solid

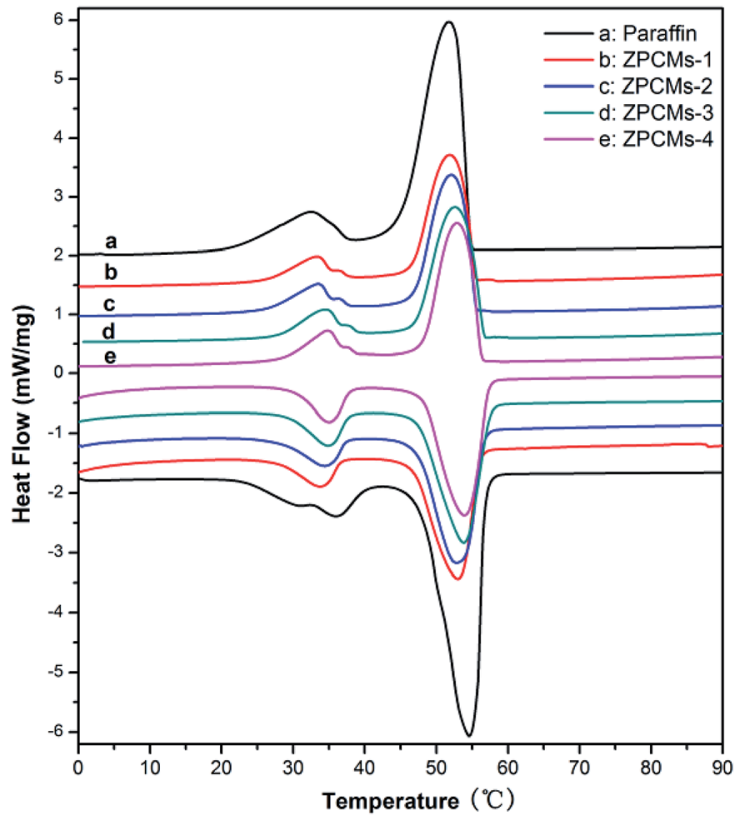

Fig. 6 DSC curves of pure paraffin and paraffin/PMMA/ZIF-67 composite PCMs.

Table 1 Phase change properties and encapsulation ratio of the paraffin/PMMA/ZIF-67 composite PCMs ${ }^{a}$

\begin{tabular}{|c|c|c|c|c|c|}
\hline \multirow[b]{2}{*}{ Samples } & \multicolumn{2}{|c|}{ Melting process } & \multicolumn{2}{|c|}{$\begin{array}{l}\text { Crystallization } \\
\text { process }\end{array}$} & \multirow{2}{*}{$\begin{array}{l}\text { Encapsulation } \\
\text { ratio (\%) }\end{array}$} \\
\hline & $\Delta H_{\mathrm{m}}\left(\mathrm{J} \mathrm{g}^{-1}\right)$ & $T_{\mathrm{m}}\left({ }^{\circ} \mathrm{C}\right)$ & $\Delta H_{\mathrm{c}}\left(\mathrm{J} \mathrm{g}^{-1}\right)$ & $T_{\mathrm{c}}\left({ }^{\circ} \mathrm{C}\right)$ & \\
\hline Paraffin & 192.67 & 54.50 & 191.39 & 51.83 & - \\
\hline ZPCMs-1 & 112.81 & 53.00 & 113.26 & 51.83 & 58.55 \\
\hline ZPCMs-2 & 106.06 & 52.33 & 107.54 & 51.97 & 55.04 \\
\hline ZPCMs-3 & 102.88 & 53.93 & 102.15 & 52.10 & 53.40 \\
\hline ZPCMs-4 & 100.59 & 53.83 & 101.66 & 51.93 & 52.21 \\
\hline
\end{tabular}

${ }^{a} \Delta H_{\mathrm{m}}$ and $\Delta H_{\mathrm{c}}$, are the enthalpy on the DSC heating and cooling curves, respectively; $T_{\mathrm{m}}$ and $T_{\mathrm{c}}$ are the peak temperature on the DSC heating and cooling curves, respectively.

products were subjected to vacuum-filtration, repeated wash with ethanol and dried in a vacuum oven at $40{ }^{\circ} \mathrm{C}$. Fig. 1 shows a brief procedure for fabrication of shape-stabilized paraffin/ PMMA/ZIF-67 composites.

Four kinds of paraffin/PMMA/ZIF-67 composites with different dosages of ZIF-67 crystals were prepared and named as ZPCMs-1 (1.0 g of ZIF-67), ZPCMs-2 (1.5 g of ZIF-67), ZPCMs-3 (2.0 $\mathrm{g}$ of ZIF-67) and ZPCMs-3 (2.5 $\mathrm{g}$ of ZIF-67).

\subsection{Measurements}

Chemical structure of paraffin/PMMA/ZIF-67 composite PCMs was estimated by a Fourier transform infrared spectrometer (FT-IR, Nicolet iS10). The crystalline structure of the samples was analyzed by X-ray diffraction (XRD) patterns using a Bruker D8 Advance diffractometer with Co $\mathrm{K} \alpha$ radiation at room temperature. Morphology and elemental composition of 

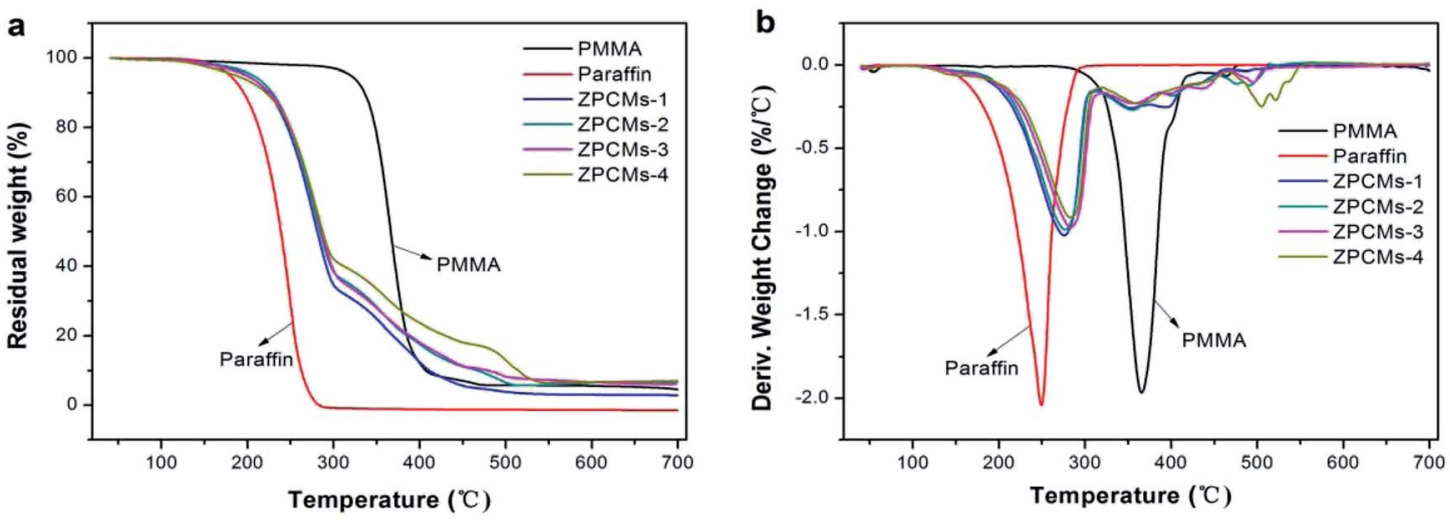

Fig. 7 TG (a) and DTG (b) curves of the pure paraffin, PMMA and various composite PCMs samples.

Table 2 The maximum decomposition temperature $\left(T_{\text {peak }}\right)$ of paraffin, PMMA and composite PCMs samples

Samples Paraffin PMMA $\quad$ ZPCMs-1 $\quad$ ZPCMs-2 $\quad$ ZPCMs-3 $\quad$ ZPCMs-4

\begin{tabular}{lllllll}
\hline$T_{\text {peak }}\left({ }^{\circ} \mathrm{C}\right)$ & 249.3 & 365.7 & 284.5 & 286.8 & 292.8 & 293.7
\end{tabular}

composites were determined by scanning electron microscopy (SEM, Hitachi S-4700) combined with an energy-dispersive X-ray spectrometer (EDX). Phase change behaviors and thermal stability of paraffin/PMMA/ZIF-67 composite PCMs were studied by differential scanning calorimetry (DSC, Q20 Ta) and a SDT Q600 thermal gravimetric analyzer (TGA) at a heating/ cooling rate of $10{ }^{\circ} \mathrm{C} \min ^{-1}$ in $\mathrm{N}_{2}$, respectively.

\section{Results and discussion}

\subsection{Structure and surface morphology}

The typical SEM images of the prepared paraffin/PMMA/ZIF-67 composite PCMs are shown in Fig. 2. It can be seen that the resultant composite PCMs exhibit an ellipse-like structure with a diameter of $10-20 \mu \mathrm{m}$.

The SEM micrographs of the composite PCMs with different contents of ZIF-67 were shown in Fig. S2 (see in ESI $\dagger$ ). As shown in Fig. S2a, $\uparrow$ the product with $1.0 \mathrm{~g}$ of ZIF-67 crystals (ZPCMs-1) presents an aggregate structure, which due to the Pickering emulsion with low content of ZIF-67 crystals had poor stability and then resulted in some unstabilized paraffin. As the ZIF-67 weight increased to $1.5 \mathrm{~g}$, the resultant composites show a desirable structure. Especially, the micrograph at high magnification (Fig. 2b) shows that the ZIF-67 crystals tightly surround on the shape-stabilized composite PCMs surface. This phenomenon attributes that the formed PMMA chains inside the oil droplets were hardly dissolved in melt paraffin. Then, these polymer chains deservedly separated from the reaction medium and deposited on the interface of ZIF-67 crystals and paraffin. Consequently, the ZIF-67 crystals were well-located on the composite PCMs surface.

However, with further increasing the weight to $2.5 \mathrm{~g}$, the mean size of composites seems to has a slightly decrease and a plenty of small size of free particles were found in products (Fig. S2d, ESI $\dagger$ ). Previous literatures have described that there exists a relationship between the particles concentration and mean droplet diameter of Pickering emulsion, and the particles are not completely covered on the droplets surface. ${ }^{27,28}$ Increasing the particles concentration will decrease the mean droplet diameter and lead to more free particles in dispersion phase. Therefore, the existence of abundant ZIF-67 crystals in Pickering emulsion system for the preparation of ZPCMs-4 resulted in the obtained products with lots of dissatisfactory morphology. The above SEM results demonstrate that the content of ZIF-67 crystals in recipe has a great effect on the morphology of paraffin/PMMA/ZIF-67 composite PCMs.

\subsection{Chemical structures and compositions}

FT-IR analysis was done to determine the formation of paraffin/ PMMA/ZIF-67 composites PCMs. Fig. 3 shows the FT-IR spectra of the ZIF-67, PMMA shell, paraffin wax and composite PCM sample. The spectrum of ZIF-67 exhibits the peaks at 1579 and $1416 \mathrm{~cm}^{-1}$ corresponding to the stretching vibration of imidazole ring, and the peaks appeared in the range of 1340$880 \mathrm{~cm}^{-1}$ and below $800 \mathrm{~cm}^{-1}$ assigning to the plane and out of plane vibration of imidazole ring, respectively. ${ }^{29,30}$ As for pure PMMA, the C-H stretching vibrations can be observed at 2997$2850 \mathrm{~cm}^{-1}$, while the peaks appearing at 1723 and $1141 \mathrm{~cm}^{-1}$ were owed to $\mathrm{C}=\mathrm{O}$ and $-\mathrm{OCH}_{3}$ stretching vibrations, respectively. As seen in the spectrum of paraffin wax, it shows the characteristic absorption peaks of in-plane rocking vibration $\left(723 \mathrm{~cm}^{-1}\right)$, deformation vibrations $\left(1379 \mathrm{~cm}^{-1}\right)$ and symmetric stretching vibration $\left(2853 \mathrm{~cm}^{-1}\right)$ of $\mathrm{CH}_{2}$. The peaks at 1460 and $2921 \mathrm{~cm}^{-1}$ belonged to deformation vibrations and symmetric stretching vibration of $\mathrm{CH}_{3}$, respectively. ${ }^{6}$ Similar distinguishable characteristic absorption peaks deriving from ZIF-67 (1304 and $\left.692 \mathrm{~cm}^{-1}\right)$, PMMA $\left(1723 \mathrm{~cm}^{-1}\right)$ and paraffin (2921, 2853, 1460 and $723 \mathrm{~cm}^{-1}$ ) are observed in the composite PCM sample, indicating that the successful preparation of paraffin/PMMA/ ZIF-67 composites PCMs.

XRD measurements were taken to confirm the crystal structure of the ZIF-67 and the composites. The results were shown in the Fig. 4. The XRD pattern of solid paraffin is in good 
accordance with the reported result. ${ }^{31}$ And the results from Fig. S1 (ESI $\dagger$ ) have confirmed that ZIF-67 was well-developed and successfully synthesized. As for composite PCMs, it shows two obvious peaks at $21.5^{\circ}$ and $23.9^{\circ}$, which assign to solid paraffin crystal, while the other main peaks at around $7.4^{\circ}$, $10.4^{\circ}, 12.8^{\circ}, 14.7^{\circ}, 16.5^{\circ}$, and $18.0^{\circ}$ belong to ZIF- 67 crystals. Besides, there are no clear diffraction peaks which may from other substances. The above results suggest that ZIF-67 and paraffin could maintain their crystal structure after being composited.

Fig. 5 shows the EDX spectrum of the composite PCMs sample (ZPCMs-3). From Fig. 5, the elemental characteristic signals for $\mathrm{C}(0.26 \mathrm{keV}), \mathrm{O}(0.53 \mathrm{keV})$ and Co $(6.9 \mathrm{keV})$ were clearly observed in the EDX spectrum. ${ }^{5,32,33}$ Since ZIF-67 was the sole source for Co element, it can be verified that ZIF-67 crystals were successfully attached on the composite surface.

\subsection{Phase change behaviors}

DSC analysis was applied to investigate the phase change behaviors of the paraffin/PMMA/ZIF-67 composite PCMs and the results are shown in Fig. 6. The characteristic parameters obtaining from DSC evaluation are presented in Table 1.

It can be seen in Fig. 6 that pure paraffin and four composite PCMs show similar DSC heating/cooling curves, despite of their great differences in terms of peak-area. The major peak at 48$65{ }^{\circ} \mathrm{C}$ corresponded to the solid-liquid transition and the minor peak appeared in the range of $35-48{ }^{\circ} \mathrm{C}$ caused by the solidsolid transition. ${ }^{6,34}$ From Table 1, we can observe that the values of $T_{\mathrm{m}}$ (melting peak temperature) for the composites reduced when compared to pure paraffin, while the values of $T_{\mathrm{c}}$ (cooling peak temperature) higher than that of pure paraffin. It is deduced that the specific surface areas of shape-stabilized PCMs are bigger than that of pure bulk paraffin. Thus, composite PCMs melt more easily than pure paraffin. During the cooling process, few ZIF-67 crystals existing in paraffin may promote the nucleating and crystallizing rate of paraffin by heterogeneous nucleation and further give rise to an increase in crystallization temperature.

The phase change enthalpies of samples were obtained by calculating the endothermic/exothermic peak-area. And then the encapsulation ratio $(E)$ of paraffin in composites was calculated by the following equation. ${ }^{35}$

$$
E=\Delta H_{\mathrm{m}, \mathrm{c}} / \Delta H_{\mathrm{m}, \mathrm{p}} \times 100 \%
$$

where $\Delta H_{\mathrm{m}, \mathrm{p}}$ and $\Delta H_{\mathrm{m}, \mathrm{c}}$ are the melting enthalpy of pure paraffin and paraffin/PMMA/ZIF-67 composite PCMs, respectively. The values of phase change enthalpies and $E$ of the composite PCMs are presented in Table 1.

As seen from Table 1, the phase-change enthalpies of the paraffin/PMMA/ZIF-67 composites reduced from that of pure paraffin, since both PMMA and ZIF-67 are inactive materials which perform no phase transformation in scanning temperature range. In addition, with respect to the encapsulation ratio of paraffin in four composite samples, it showed that the values of $E$ increased with the increase of ZIF-67 weight. There is no doubt that increasing the weight of ZIF-67 in recipe will result the actual percentage of ZIF-67 in resultant composites increased, then the encapsulation ratio of paraffin correspondingly decreased. Combining with the SEM results about the morphology of composite with various weights of ZIF-67 crystals, the composite PCMs with favourable morphology and extremely phase change enthalpy of $106.06 \mathrm{~J} \mathrm{~g}^{-1}$ could be achieved by the sample prepared with $1.5 \mathrm{~g}$ of ZIF-67.

\subsection{Thermal stability}

The thermal stability of PCMs is crucial to assess their applications in heat energy storage. ${ }^{36}$ TG analysis was used to investigate the thermal degradation property of the developed paraffin/PMMA/ZIF-67 composite PCMs and the results are shown in Fig. 7. From Fig. 7, we can see that pure paraffin exhibits a marked one-step weight loss in the range of $110^{\circ} \mathrm{C}$ to $300{ }^{\circ} \mathrm{C}$ with almost no char remained, demonstrating that paraffin underwent a simple evaporation. ${ }^{37}$ The TG/DTG thermogram of PMMA indicates a degradation step between $300{ }^{\circ} \mathrm{C}$ and $420{ }^{\circ} \mathrm{C}$ due to the thermal decomposition of polymer chains. Compared to pure paraffin and PMMA, the paraffin/ PMMA/ZIF-67 composite samples all exhibit distinguishable three-step degradation in their TG curves. The decomposition peaks appeared in the range of $115-316{ }^{\circ} \mathrm{C}$ and $317-460{ }^{\circ} \mathrm{C}$ corresponded to the evaporation of paraffin and PMMA pyrolysis, respectively, while the slight degradation step was observed at $420-560{ }^{\circ} \mathrm{C}$ attributing to the decomposition of ZIF67 crystals.

The maximum decomposition temperature $\left(T_{\text {peak }}\right)$ is summarized in Table 2 . It is obviously observed that the thermal decomposition temperature of the shape-stabilized composites is higher than that of pure paraffin (the first degradation step). As shown in Table 2, the $T_{\text {peak }}$ for ZPCMs- 1 is $35.2^{\circ} \mathrm{C}$, which higher than that of pure paraffin. Moreover, the values of $T_{\text {peak }}$ increased with the increase of ZIF-67 crystals content. Such a considerable increase of $T_{\text {peak }}$ for composite PCMs was ascribed to the formation of compact PMMA/ZIF-67 composite shell on the paraffin surface. The composites can availably block the flow of thermal transmission and slowly decrease the propagation or evaporation of the solid paraffin to the gas phase. ${ }^{38}$ Therefore, it can be inferred that the PMMA/ZIF-67 composites effectively served as a protective layer for the paraffin core, and the increase of ZIF-67 loading made the composite shells stronger due to the excellent thermal stability of ZIF-67 crystals.

\section{Conclusion}

In the present work, a novel shape-stabilized paraffin/PMMA/ ZIF-67 composite PCMs with approximate sphere-like structure was successfully prepared by using a Pickering emulsion route, in which ZIF-67 crystals were served as stabilizers. The loading content of ZIF-67 crystals in emulsion great affects the morphology of the composite PCMs. When adding $1.5 \mathrm{~g}$ of ZIF67 crystals in recipe, the obtained composite PCMs appear spherical shape with abundant ZIF-67 crystals well-arranged on the composites surface, and the corresponding composite 
PCMs have about $106 \mathrm{~J} \mathrm{~g}^{-1}$ of latent heat. TGA results showed that the composite PCMs exhibited higher maximum decomposition temperature of ZPCMs-1 than that of pure paraffin, because ZIF-67 crystals with excellent thermal stability were highly introduced in the composites and served as a protective layer for the paraffin core. The composite PCMs prepared in this work show a good potential application in thermal energy storage technology.

\section{Conflicts of interest}

There are no conflicts to declare.

\section{Acknowledgements}

The authors thank Dr Sun for her helpful discussions.

\section{References}

1 Y. Wang, B. Tang and S. Zhang, RSC Adv., 2012, 2, 5964.

2 N. P. Sharifi, A. A. N. Shaikh and A. R. Sakulich, Energy Build., 2017, 138, 455.

3 N. Soares, J. J. Costa, A. R. Gaspar, et al., Energy Build., 2013, $59,82$.

4 Q. Tang, J. Sun, S. Yu, et al., RSC Adv., 2014, 4, 36584.

5 B. Xu, H. Ma, Z. Lu, et al., Appl. Energy, 2015, 160, 358.

6 N. Sun and Z. Xiao, J. Mater. Sci., 2016, 51, 8550.

7 D. Yang, S. Shi, L. Xiong, et al., Sol. Energy Mater. Sol. Cells, 2016, 144, 228.

8 W. Chalco-Sandoval, M. J. Fabra, A. López-Rubio, et al., Eur. Polym. J., 2015, 72, 23.

9 X. Li, J. G. Sanjayan and J. L. Wilson, Energy Build., 2014, 76, 284.

10 R. Al-Shannaq, M. Farid, S. Al-Muhtaseb, et al., Sol. Energy Mater. Sol. Cells, 2015, 132, 311.

11 E. M. Fayyad, M. A. Almaadeed and A. Jones, Polym. Bull., 2016, 73, 631.

12 F. Jeremias, D. Fröhlich, C. Janiak, et al., RSC Adv., 2014, 4, 24073.
13 H. Furukawa, K. E. Cordova, M. O'Keeffe, et al., Science, 2013, 341, 1230444.

14 B. Wang, X. L. Lv, D. Feng, et al., J. Am. Chem. Soc., 2016, 138, 6204.

15 G. C. Shearer, S. Chavan, S. Bordiga, et al., Chem. Mater., 2016, 28, 3749.

16 M. Zhao, K. Yuan, Y. Wang, et al., Nature, 2016, 539, 76.

17 K. Zhou, B. Mousavi, Z. Luo, et al., J. Mater. Chem. A, 2017, 5, 952.

18 X. Li, X. Gao, L. Ai, et al., Chem. Eng. J., 2015, 274, 238.

19 R. Aveyard, B. P. Binks and J. H. Clint, Adv. Colloid Interface Sci., 2013, 100, 503.

20 B. P. Binks and S. O. Lumsdon, Langmuir, 2001, 17, 4540.

21 J. Huo, M. Marcello, A. Garai, et al., Adv. Mater., 2013, 25, 2717.

22 Z. Li, J. Zhang, T. Luo, et al., Soft Matter, 2016, 12, 8841.

23 R. Sabouni and H. Gomaa, Soft Matter, 2015, 11, 4507.

24 R. Li, J. Chen, X. Zhou, et al., RSC Adv., 2016, 6, 102534.

25 R. Liang, Y. Jiang, W. Yokoyama, et al., RSC Adv., 2016, 6, 99496.

26 Y. Pan, H. Li, X. X. Zhang, et al., Chem. Eng. Sci., 2015, 137, 504.

27 B. P. Binks and J. H. Clint, Langmuir, 2018, 18, 1270.

28 K. Zhang, W. Wu, K. Guo, et al., Colloids Surf., A, 2009, 349, 110.

29 K. Y. A. Lin, H. Yang and W. D. Lee, RSC Adv., 2015, 5, 81330. 30 K. Vellingiri, P. Kumar, A. Deep, et al., Chem. Eng. J., 2017, 307, 1116.

31 C. Li, L. Fu, J. Ouyang, et al., Appl. Clay Sci., 2015, 115, 212. 32 X. Zhang, G. Ji, W. Liu, et al., J. Mater. Chem. C, 2016, 4, 1860.

33 J. Zhao, Y. Yang, Y. Li, et al., Sol. Energy Mater. Sol. Cells, 2017, 168, 62.

34 W. Liang, G. Zhang, H. Sun, et al., RSC Adv., 2013, 3, 18022.

35 C. Alkan, A. Sarı and A. Karaipekli, Sol. Energy Mater. Sol. Cells, 2011, 52, 687-692.

36 L. Miró, C. Barreneche, G. Ferrer, et al., Thermochim. Acta, 2016, 627, 39.

37 X. Jiang, R. Luo, F. Peng, et al., Appl. Energy, 2015, 137, 731.

38 C. Li, L. Fu, J. Ouyang, et al., Appl. Clay Sci., 2015, 115, 212. 